\title{
Die Bedeutung des Habitus-Konzepts für die Erforschung soziokultureller Unterschiede im Bereich der Medienpädagogik
}

Ralf Biermann

\begin{abstract}
In diesem Artikel geht es um die nähere Betrachtung, welche grundlegenden Schnittstellen sich zwischen medienpädagogischen Ansätzen und der HabitusTheorie von Pierre Bourdieu ergeben. Basierend auf diesen Überlegungen werden Aspekte ausgearbeitet, die in medienpädagogischer Forschung und Praxis zur Beachtung soziokultureller Unterschiede Berücksichtigung finden können.
\end{abstract}

\section{Die Existenz soziokultureller Unterschiede}

Das Habitus-Konzept und die ihm zu Grunde liegenden umfangreichen Arbeiten Bourdieus (1979, 1982, 1987, 1997) erfahren zurzeit (wieder) eine grosse Aufmerksamkeit. Dies liegt unter anderem an dessen Erklärungskraft und damit zusammenhängend an der empirischen Nutzungsmöglichkeit zur Erarbeitung von soziokulturellen Unterschieden. Diese Differenzen zwischen Individuen und zwischen Gruppen (gemessen an der unterschiedlichen Kapitalausstattung und die damit verbundenen Chancen auf Bildung etc.) stehen zunehmend im Blickpunkt des öffentlichen Interesses, vor allem durch die von den PISA-Studien initiierten Diskussionen und der damit einhergegangenen Ernüchterung, dass der schulische Erfolg in Deutschland in einem hohen Masse von der sozialen Herkunft abhängt (vgl. Deutsches PISA-Konsortium 2005). Der sozioökonomische Status der Familie gilt dabei als entscheidender Faktor für den Schulerfolg. Gerade die (Schul-)Pädagogik mit ihrem anerkennenswerten Ziel, allen Heranwachsenden (in Abhängigkeit ihrer scheinbar objektiv bewertbaren Leistungen) gleiche Chancen auf Bildung und somit auf adäquate berufliche Aussichten und gesellschaftlicher Anerkennung sicher zu stellen, fördert - so die empirischen Befunde - ganz im Sinne Bourdieus die Ungleichheit anstatt sie abzubauen (vgl. Bourdieu 2006, S.25-52 sowie Ditton 2007).

Dies kann durchaus als Provokation an die Pädagogik verstanden werden, die damit in ihren fundamentalen Annahmen und der Legitimation der eigenen Existenz in Frage gestellt werden kann (vgl. hierzu die Ausführungen von Liebau 2006). Auch in der erziehungswissenschaftlichen Diskussion lässt sich eine verstärkte auf 
das Werk Bourdieus gerichtete Aufmerksamkeit feststellen (vgl. exemplarisch den Sammelband von Friebertshäuser et. al. 2006).

Das Habitus-Konzept ist in vielfältiger Weise an pädagogische Diskurse anschlussfähig, indem es z. B. bei der Integration zielgruppenspezifischer Perspektiven in pädagogische Konzepte hilft. Da es zudem als Grundlage für die Beantwortung von Fragen zur Genese benachteiligender soziokultureller Unterschiede dienen kann, bietet es zugleich die Möglichkeit darauf rekurrierend Lösungsansätze für pädagogische Massnahmen zu entwickeln und methodische Forschungszugänge zu konkretisieren.

Habituelle Differenzen existieren auch im Bereich der Mediennutzung: «Befunde der Medienforschung belegen, dass Medien nicht die grossen Gleichmacher sind, sondern sehr unterschiedlich genutzt werden» (Niesyto 2007, S. 153). Bei der Betrachtung der Mediennutzung von Kindern und Jugendlichen (vgl. MPFS 2007a und 2007b) werden zunächst geschlechtsspezifische Unterschiede deutlich. Bereits während des Kindesalters beginnen sich unterschiedliche Vorlieben in der Mediennutzung auszubilden: Im Bereich Fernsehen, Bücher lesen und Computer(spiele) finden sich erste - teilweise sehr starke - Differenzen in den Nutzungsmustern. Diese verstärken sich bzw. weiten sich meist noch während des Jugendalters weiter aus. So finden sich Unterschiede beim Lesen von Büchern (vgl. MPFS 2007b, S. 21) wie auch beim liebsten Fernsehprogramm (vgl. ders., S. 24) und den liebsten Fernsehsendungen (vgl. ders., S. 27). Besonders eklatant sind die Ungleichheiten bei der Computer- und der Internetnutzung, bei der männliche Probanden z. B. eine deutlich höhere Affinität bezogen auf die Bindung und Nutzung zu Computerspielen aufweisen (vgl. ders., S. 31ff).

Auch im Hinblick auf das Bildungsmilieu finden sich deutliche Differenzen: «Hauptschüler nennen häufiger den Fernseher (18\%) als Realschüler (16\%) und Gymnasiasten (13\%)» (MPFS 2007a, S. 17) als unverzichtbares Medium. Beim Internet kehrt sich dieser Sachverhalt um. Mit höherer Schulbildung geben die Schüler deutlich öfter an, dass sie nicht auf das Internet verzichten könnten (vgl. ders., S. 17). «Unter dem Aspekt des Bildungshintergrundes der Jugendlichen zeigen Gymnasiasten insgesamt das breiteste Anwendungsspektrum. Sie nutzen Instant Messenger und E-Mails häufiger und setzen das Internet sehr viel stärker für Recherche und Information ein als Real- und Hauptschüler» (ders. S. 40). Ebenso finden sich Differenzen beim Lesen (vgl. ders., S. 21) und im geringeren Masse beim Fernsehen (vgl. ders, S. 24).

Diese hier beispielhaft aufgeführten quantitativ gewonnenen Ergebnisse machen vor allem deutlich, dass sich Unterschiede in vielfältiger Weise bemerkbar machen. Allerdings zeigen sie nicht, wie diese sich reproduzieren bzw. aufgrund welcher Existenzbedingungen diese Differenzen zustande kommen. Betrachtet man die alterspezifischen Unterschiede zwischen den beiden Studien des MPFS, so fällt auf, dass sich die Abweichungen verstärken und ausweiten, also im Verlauf des Soziali- 
sationsprozesses verfestigen. Zwar lassen sich dabei Faktoren wie das Geschlecht, der Bildungshintergrund etc. als klassifizierende Merkmale zur Unterscheidung der Mediennutzung ausmachen, dennoch bleibt hierbei unklar, wie stark diese jeweils zur Genese der Differenzen beitragen. Deutlicher wird der Zusammenhang zwischen Bildungshintergrund und der Mediennutzung bei den Studien des Kompetenzzentrums Informelle Bildung der Universität Bielefeld: «Die Ergebnisse der qualitativen und quantitativen Erhebungen zu Nutzungsweisen und Aneignungsstrukturen von Jugendlichen im Internet zeigen deutlich, dass die soziokulturellen Bedingungen des ‘Offline-Leben` gravierende Auswirkungen auf die Onlinenutzung haben. Insbesondere der Bildungshintergrund der Jugendlichen sowie ihrer sozialen Umgebung spielt eine zentrale Rolle» (Otto et al. 2005, S. 3. Herv. Im Original). An dieser Aussage wird deutlich, wie die unterschiedlichen Teilbereiche des Alltags miteinander verwoben sind und individuelle Tendenzen von Handlungen beeinflussen.

Das Habitus-Konzept kann als Bezugsrahmen für medienpädagogische Arbeiten (in Forschung und Praxis) dienen, der für das Verstehen der Genese und Reproduktion der soziokulturellen Unterschiede der Mediennutzung hilfreich ist.

Im Folgenden soll zunächst kurz geklärt werden, inwiefern sich das Habitus-Konzept mit medienpädagogischen Ansätzen verbinden lässt und zu einem breiteren Verständnis soziokultureller Unterschiede - besonders im Bereich der Medien beitragen kann. Hierzu wird als Erstes geprüft, ob der Grundgedanke des HabitusKonzepts und mit ihm verbunden wichtige Implikationen wie die Kapitalsorten zum zentralen Begriff der Medienkompetenz kompatibel bzw. anschlussfähig erscheinen. Daran anschliessend wird gefragt, wie distinktive Muster der Bewertung (Gegensatzpaare) als Basis für die differenten Dispositionen fungieren und wie diese für eine empirische Untersuchung miteinander verwoben werden sollten.

\section{Die Verbindung des Habitus Konzepts mit medienpädagogischen Ansätzen}

In der Medienpädagogik nimmt der Begriff der Medienkompetenz und darin eingeschlossen der Kompetenz selbst eine zentrale Rolle ein. Etliche Autoren haben in diversen Publikationen ihre je eigene Definition von Medienkompetenz festgehalten (vgl. Schorb 1997, Aufenanger 1999a, Groeben 2002). Die wohl wichtigste Definition - auch weil viele andere darauf aufbauen - ist die Dimensionierung von Dieter Baacke mit den vier Aspekten Medienkunde, Mediengestaltung, Mediennutzung und Medienkritik (vgl. Baacke 1999, S. 34). Der ursprünglich von vielen medienpädagogischen Autoren verwendete und dabei durchaus weit gefasste Begriff der Medienkompetenz wird jedoch des Öfteren verkürzt dargestellt (vgl. hierzu die Sammlung von Definitionen von Medienkompetenz bei Gapski 2001, S. 255-293) und auch in vielfältiger Weise kritisiert (vgl. Moser 2003 und Aufenanger 1999b). Dabei ist es notwendig, sich klar zu machen, dass eine verkürzte Verwendung des Begriffs Medienkompetenz ( z. B. auf Bedienwissen) in Verbindung mit dem Habi- 
tuskonzept keinen Nutzen zur Erarbeitung soziokultureller Unterschiede bringt, da erst eine Verbindung mit den Existenzbedingungen, der Kapitalausstattung und letztendlich mit dem individuellen (medialen) Habitus Klarheit über die Motive und die Beweggründe hinter den reinen Nutzungsdaten schafft. Aus diesem Grund kann nur ein breit gefasster Begriff der Medienkompetenz Verwendung finden.

Ein immer wieder angeführter Kritikpunkt ist der Rekurs von Baacke auf Chomsky (vgl. Baacke 1973, S. $100 \mathrm{f}$ und 262f), mit dem er versucht hat, den Menschen als kompetentes Lebewesen darzustellen und die kreativen Akte der Kommunikation zu begreifen. Die von Chomsky beschriebene generative Grammatik ermöglicht es Individuen, überaus flexibel auf verschiedene (Sprach-)Situationen zu reagieren und eine unendliche Anzahl von Sätzen zu generieren, auch wenn sie diese noch nie gehört haben. Diesen Rekurs auf Chomsky nimmt auch Bourdieu als Erklärung der generativen Strukturen des Habitus vor, um damit dessen schöpferischeres Potential zu verdeutlichen (vgl. Krais/Gebauer 2002, S. 31). Im Gegensatz zu Baacke (1999, S. 33) distanziert sich Bourdieu jedoch bereits zugleich von der Annahme Chomskys, dass die generative Grammatik angeboren sei und verweist auf die erfahrungsabhängige Konstruktion des Habitus (vgl. Krais/Gebauer 2002, S. 31).

Genau an diesem Punkt bezieht sich Baacke dann auf Bourdieu. Das folgende längere Zitat soll die Gemeinsamkeiten in den Argumentationslinien Bourdieus und Baackes zur Entwicklung der beiden Konzepte «Habitus〉 und 'Medienkompetenz〉 und den gemeinsamen Blick auf soziokulturelle Unterschiede im Handeln von Akteuren aufzeigen:

Bourdieu bezog sich wie Habermas (und Baacke in seiner Schrift «Kommunikation und Kompetenz〉 1972) auf Chomskys Begriff und Konzept der «Kompetenz». Bourdieu betonte freilich stärker die «generative Grammatik» und verstand «Habitus» als ein System von Mustern, die der Mensch verinnerlicht hat und die es ihm ermöglichen, variabel Wahrnehmungen, Gedanken und auch Handlungen eines kulturellen Raums zu erzeugen. In der generativen Grammatik sind alle Sätze virtuell beschlossen, die ein Mensch äussern und umsetzen kann, und im Habitus kommen sie gleichsam an die Oberfläche. Bourdieu meinte, durchaus marxistisch, dass der Habitus nicht frei verfügbar sei, sondern der Mensch je nach sozialer Klassen- und Schichtzugehörigkeit sich jene Deutungsmuster zuordne, die nach dem zugeteilten «Bildungskapital» für ihn zugänglich seien. Die soziale Herkunft und die Schulbildung sind für Bourdieu also von grosser Bedeutung, um einen variantenreichen Habitus auszubilden und damit die ‘Kompetenz〉 des Menschen vollständiger zu entfalten. Er sieht das kulturelle Kapital an kommunikativer Kompetenz auf verschiedene soziale Milieus und Klassen verteilt und zwar ungleich.

Dieser soziologische Diskurs ist in vielfältiger Hinsicht anschlussfähig. Um nur ein Beispiel zu geben: Die Wissenskluft-Hypothese (knowledge gap hypothesis), wonach die Nutzung neuer Medien nur von einem eher bildungs- 
starken Teil produktiv genutzt werde, während ein anderer Teil die neuen Medien-Kombinationen monoton und unkreativ verwende, zeigt deutlich, dass «Medienkompetenz` weder ein angeborenes Muster noch ein entwicklungslogisches Muss darstellt, sondern dass die Plastizität des Habitus in seiner Ausformung wiederum abhängig ist von der Forderung durch Erziehungs- und Bildungsinstitutionen. (Baacke 1999, S. 33)

Die von Baacke postulierte vielfältige Anschlussfähigkeit an das Konzept von Bourdieu stellt eine Verbindung her, die ein umfangreiches Konzept von Medienkompetenz (vgl. Baacke 1999, S. 33) oder auch Medienbildung (vgl. Marotzki/Jörissen 2008) als inkorporierte Dispositionen (auch habituelle Muster) beschreiben lassen. Das würde bedeuten, dass der mediale Habitus unter dem Einfluss der Existenzbedingungen ein System von Grenzen ausbildet (vgl. Bourdieu 1987, S. 102-103), das zugleich als Grund für die für die unterschiedlichen Umgangsformen mit Medien und deren Reproduktion konstitutiv ist:

Der Habitus fungiert als ein handlungsermöglichendes System von Grenzen. Begrenzt oder eingegrenzt werden die Dispositionen sozialer Akteure durch die Perspektivität und Selektivität lagespezifischer Erfahrungs-, Aneignungs- und Kompetenzmuster. [...] Die Dispositionsstruktur bietet Schablonen kognitiver Realitätsverarbeitung an, d. h. praktische Wahrnehmungsund Beurteilungsschemata, die sich mit den Herkunftsbedingungen in einer «habituellen Komplizenschaft» befinden. (Bauer 2002)

Für die weiteren Erläuterungen erscheint es zunächst notwendig, die Funktionsweise des Habitus und die damit verbundene Ausbildung des (sozialen und kulturellen) Kapitals zu explizieren.

\section{Die Funktionsweise des Habitus}

Die ersten Arbeiten von Bourdieu zum Begriff des Habitus gehen auf seine Zeit in Algerien zurück. Er untersuchte das Verhalten der Kabylen, das seine Grundlage in den durch die Lebensverhältnisse geprägten Erfahrungen hatte (vgl. Bourdieu 2003). «Die Erfahrung dieser Existenzbedingungen hatte sich in bestimmten Wahrnehmungs- und Handlungs-Dispositionen in den Individuen niedergeschlagen, die selbst dann noch wirksam waren, als ihre materiellen Lebensverhältnisse sich tief greifend verändert hatten» (Krais/Gebauer 2002, S. 22. Dies bedeutet zum einen, dass die Lebens- und Existenzbedingungen massgeblichen Einfluss auf die lebenslange Entwicklung und Ausformung des Habitus haben, zum anderen, dass der Habitus auf Basis der gemachten Erfahrungen Handlungspraxen reproduziert (wenn auch mit Abweichungen zu vorherigen). Damit eröffneten sich Bourdieu die zwei Seiten des Habitus: «Der Habitus ist nicht nur strukturierende, die Praxis wie deren Wahrnehmung organisierende Struktur, sondern auch strukturierte Struktur: das Prinzip der Teilung in logische Klassen, das der Wahrnehmung der sozialen 
Welt zugrunde liegt, ist einerseits Produkt der Verinnerlichung der Teilung in soziale Klassen» (Bourdieu 1982, S. 279).

«Als System generativer Schemata von Praxis» (Bourdieu 1982, S. 279) trägt der Habitus als Erzeugungssystem dazu bei, klassifizierbare Praxisformen und Werke hervorzubringen und eine Unterscheidung und Bewertung der Formen und Produkte (Geschmack) zu ermöglichen. Darin repräsentiert sich die soziale Welt, der Raum der Lebensstile (vgl. Bourdieu 1982, S. 277-278).

In den Dispositionen des Habitus ist somit die gesamte Struktur des Systems der Existenzbedingungen angelegt, so wie diese sich in der Erfahrung einer besonderen sozialen Lage mit einer bestimmten Position innerhalb dieser Struktur niederschlägt. Die fundamentalen Gegensatzpaare der Struktur der Existenzbedingungen (oben/unten, reich/arm, etc.) setzen sich tendenziell als grundlegende Strukturierungsprinzipien der Praxisformen wie deren Wahrnehmung durch. (Bourdieu 1982, S. 279)

Der Habitus ist gleichsam generatives Prinzip und die Präsenz der Vergangenheit als inkorporierte Erfahrungen, die bei den Menschen auf unterschiedliche Existenzbedingungen rekurrieren:

Durch transformierende Verinnerlichung der äusseren (klassenspezifisch verteilten) materiellen und kulturellen Existenzbedingungen entstanden, stellt der Habitus ein dauerhaft wirksames System von (klassenspezifischen) Wahrnehmungs-, Denk- und Handlungsschemata dar, das sowohl den Praxisformen sozialer Akteure als auch den mit dieser Praxis verbundenen alltäglichen Wahrnehmungen konstitutiv zugrunde liegt. (Schwingel 2005, S. 73)

Bourdieu geht in seinen Arbeiten davon aus, dass die Gesellschaft in Klassen unterteilt ist, die unterschiedliche Chancen auf Macht und Kapital haben. Eine Definition bzw. eine genauere Beschreibung der Klassen sucht man bei Bourdieu vergeblich: «An keiner Stelle jedoch gibt Bourdieu eine systematische Darstellung der Struktur dieser Ungleichverteilung. Er spricht vom Kleinbürgertum als einer Klasse, von den Bauern, von den «classes populaires», von der herrschenden Klasse und der herrschenden Fraktion der herrschenden Klasse, ohne diese vorgängig gegeneinander abzugrenzen; vielmehr greift er auf, was im alltäglichen Sprachgebrauch als Klasse gilt» (Krais 1983, S. 215).

Die individuelle Kapitalausstattung hängt dabei in einem hohen Masse von der Gruppenzugehörigkeit ab. Das bedeutet allerdings nicht, dass mit dieser die Handlungspraxen eines Akteurs determiniert sind:

Der Habitus steht nicht - entgegen aller hartnäckigen Missverständnisse in der anfänglichen Bourdieu-Rezeption - für ein deterministisches Prinzip der sozialen Reproduktion. [...] Formen der aktiven produktiven Realitätsverarbeitung, sogar Prozesse der Selbstsozialisation nehmen in Bourdieus Habitusmodell eine grundlegende Bedeutung ein. Ohne sie ist weder soziale 
noch individuelle Praxis denkbar. (Bauer 2002, S. 136 und in ähnlicher Form Fuchs-Heinritz/König 2005, S. 130-131)

Der oben vorgestellte Rekurs auf die Wissenskluft-Hypothese in Verbindung mit «bildungsstarken» Nutzungsmustern lässt sich ohne grosse Probleme mit der Habitus-Theorie koppeln. Bourdieu zeigt in seinen Arbeiten auf, wie gesellschaftliche Ungleichheiten aufgrund unterschiedlicher Existenzbedingungen entstehen und wie diese durch Reproduktion und Trägheitseffekte (Hysteresis) aufrechterhalten werden. Diese Differenzen bestehen somit aufrund der ungleichen Verteilung von Kapital, das über individuelle (und - auch betrachtet im Rahmen des Herkunftsmilieus - gruppenspezifische) Erfahrungen akkumuliert wird. Die Ausstattung stellt damit eine grundlegende Komponente des Habitus dar. Die Differenzierung in unterschiedliche Ausstattungsmerkmale geht über den von Marx verwendeten Begriff des Kapitals hinaus, der sich auf den Besitz von Produktionsmitteln bezieht, indem er soziale und kulturelle Kenntnisse, Fähigkeiten und Fertigkeiten als gesonderte Kapitalsorten mit einbezieht.

\section{Die Kapitalausstattung als Beschreibung der spezifischen Dispositionen eines Akteurs}

\section{Das ökonomische Kapital}

Ganz bewusst steht das ökonomische Kapital wegen seiner grundlegenden Funktion an erster Stelle. "Zum ökonomischen Kapital zählen alle Formen des materiellen Besitzes, die in Gesellschaften mit einem entwickelten Markt in und mittels Geld getauscht werden können» (Fuchs-Heinritz/ König 2005, S. 161). Aus der Sicht Bourdieus erscheint das ökonomische Kapital als grundlegend (vgl. Baumgart 2000, S. 227 sowie Fuchs-Heinritz/König 2005, S. 161). Da die anderen Kapitalsorten (s. u.) unter Einbezug eines Transformationsprozesses aus dem Einsatz von ökonomischem Kapital entstehen bzw. akkumuliert werden können und eine Zurückführung auf das ökonomische niemals ganz möglich ist, kommt diesem Kapital eine besondere Rolle zu (vgl. Baumgart 2000, S. 226-227).

Natürlich dient das ökonomische Kapital nicht nur dazu, andere Kapitalsorten zu akkumulieren, sondern auch dem Konsum, also der Anschaffung von Medien(geräten). Eine Mediennutzung ist immer mit Kosten verbunden, sei es in der Anschaffung und/oder im Betrieb durch laufende Kosten und müssen zunächst über das Einkommen erwirtschaftet werden, vor allem dann, wenn keine Reserven einen Ausfall des regelmässigen Einkommens kompensieren können. ${ }^{1}$ Bezogen auf die Medien verschiebt sich die Diskussion vom Digital Divide (Zugang zu Medien, auch unter ökonomischen Aspekten) zum Participatory Divide, bei dem es um

Als Beispiel kann die Diskussion zu den Studiengebühren herangezogen werden. Zu wenig ökonomisches Kapital und/oder zu hohe Kosten können durchaus das Studium beeinträchtigen (notwendige Nebenjobs etc.). 
die Teilhabe an Kommunikation und den Umgang mit Medien geht. Hierbei steht verstärkt das kulturelle Kapital im Vordergrund.

\section{Das kulturelle Kapital}

Das kulturelle Kapital (vgl. Bourdieu 2006, S. 112-120) existiert in drei Formen. Als inkorporierte und somit körpergebundene Kultur, «auf deutsch Bildung» (Bourdieu 1983, S. 185), besteht «kulturelles Kapital aus den kulturellen Kenntnissen, Fähigkeiten und Fertigkeiten eines Individuums [...]. In dieser Form ist kulturelles Kapital weder durch Geld zu erwerben noch direkt in Geld zu konvertieren. Erworben wird es durch persönliche (Bildungs-) Bemühungen» (Fuchs-Heinritz/König 2005, S. 163). Dieser Erwerb kostet Zeit, in der kein ökonomisches Kapital akkumuliert werden kann. Dies zeigt sich z. B. bei dem Erwerb von Hochschulabschlüssen im Vergleich zu Berufsausbildungen, die Haupt- und Realschulabschlüsse voraussetzen. Während über das Abitur und das darauf folgende Studium kulturelles Kapital angehäuft wird und i. d. R. keine umfangreiche Anstellung möglich ist, verdient eine Person mit einer Berufsausbildung früher Geld, fördert damit aber nicht den intensiven Aufbau von kulturellem Kapital. Die Ausformung von kulturellem Kapital ist somit mit Anstrengungen verbunden und - wie im Falle des inkorporierten und institutionalisierten Kapitals (s. u.) - an die Person gebunden.

Die Übertragung des kulturellen Kapitals von den Eltern auf deren Kinder und der Zusammenhang mit den schulischen Leistungen machen die Bedeutung des familialen Milieus ${ }^{2}$ deutlich (vgl. Bourdieu 2006, S. 26-31). Die Kinder inkorporieren das von den Eltern vorgelebte kulturelle Kapital und reproduzieren auf diese Weise in ihren späteren Handlungen. Dabei ist die Besonderheit des inkorporierten Kapitals zu beachten: Die angeeigneten Kenntnisse, Fähigkeiten und Fertigkeiten sind kein Besitztum das veräussert werden kann, sie werden «zu einem festen Bestandteil der 〈Person», zum Habitus [...]; aus «Haben» ist «Sein» geworden» (Baumgart 2000, S. 220). Dabei ist jedoch anzumerken, dass es keine $100 \%$ ige Übertragung gibt, d.h., die Reproduktion selbst unterliegt Veränderungen. Jedoch finden diese in einem Rahmen statt, der eine Verbindung zu den bisherigen Mustern der Eltern aufzeigt. Dieses lässt sich auf das Medienhandeln anwenden. So zeigen sich in Abhängigkeit des Herkunftsmilieus durchaus differente Erziehungsmuster und Vorbildfunktionen von Eltern und daraus resultierende entsprechende Mediennutzungsweisen von deren Kindern (vgl. Kommer/Biermann 2006, S. 210ff).

Dem inkorporierten steht das objektivierte kulturelle Kapital gegenüber: Es ist durch seine Gegenständlichkeit materiell übertragbar, z. B. Gemälde, Schriftstücke wie Bücher, Instrumente aber auch eben Filme (beispielsweise auf DVD) etc. In

2 Dabei wird durch den Wandel der Familienformen an der Wirksamkeit und Bedeutung familialer Weitergabe habitueller Muster gezweifelt (vgl. Fuchs-Heinritz/König 2005, S. 138-139). Dabei bleiben jedoch Machtverhältnisse innerhalb der Familie und die Schwierigkeit aus den eigenen MilieuGrenzen auszubrechen unberücksichtigt. 
dieser Eigenschaft unterscheidet es sich fundamental von dem inkorporierten und institutionalisierten Kapital (s. u.), wobei zu beachten ist, dass lediglich der Besitz übertragen werden kann. So ist es ohne weiteres möglich, ein Gemälde zu erwerben, jedoch nicht die «Verfügung über kulturelle Fähigkeiten, die den Genuss des Gemäldes [...] erst ermöglichen» (Baumgart 2000, S. 222 und Bourdieu 1983, S. 188). Die Transformation in ökonomisches Kapital ist in einfacher Form durch den Verkauf der Objekte möglich, die Übertragung der kulturellen Fähigkeiten zum Genuss dagegen ist auf ein inkorporiertes kulturelles Kapital angewiesen (vgl. Bourdieu 2006, S. 117-118). Hier liegt ein Problem pädagogischer normativer Intervention zum «richtigen» Umgang mit Medien vor, wenn die spezifischen Erfahrungen und Erwartungen von Zielgruppen unzureichend berücksichtigt oder gar ignoriert werden.

Das institutionalisierte Kapital bescheinigt dem Individuum über Zeugnisse, Zertifikate (Bildungstitel) etc. Träger von bestimmten kulturellen Kenntnissen, Fähigkeiten und Fertigkeiten zu sein, die jedoch an die Person gebunden sind. Die schriftlichen Bestätigungen sind nicht auf andere übertragbar bzw. veräusserbar. Im krassen Unterschied zu den Inhabern eines kulturellen Kapitals ohne schulische Beglaubigung, denen man immer abverlangen kann, den Beweis für ihre Fähigkeiten anzutreten, da sie nur sind was sie tun, schlichte Produkte ihrer kulturellen Leistung, brauchen die Inhaber von Bildungspatenten [...] nur zu sein, was sie sind, da doch der Wert ihrer Handlungen und Taten sich einzig und allein am Wert ihrer selbst, deren Urheber, bemisst. (Bourdieu 1982, S. 48-49)

Dies hat bedeutsame Auswirkungen auf die Berufsausübung:

Die Zulassung zu Berufen und somit die Möglichkeit das erworbene kulturelle Kapital in ein finanzielles Einkommen, d. h. in ökonomisches Kapital umzuwandeln, ist zuallererst von der Verfügung über entsprechende Legitimitätsnachweise in Form von Schul-, Berufs- und Bildungsabschlüssen abhängig. (Schwingel 2005, S. 90-91)

Damit zeigt sich die entscheidende Relevanz der beruflichen Ausbildung für das Individuum und die Verknüpfung mit der Reproduktion habitueller Muster in der Familie. «[...] Weil die Schulinstitution zwar mit dem Monopol der Zertifikation ausgerüstet ist [...], aber nicht über das Monopol der Produktion des kulturellen Kapitals verfügt» (Bourdieu 1982, S. 143), ist das 'geerbte> (vgl. Bourdieu 1982, S. 143-150) inkorporierte kulturelle Kapital von besonderer Bedeutung. Durch die Fortschreibung bzw. Reproduktion elterlicher Bildungsmilieus bei ihren Kindern und der teilweisen Umwandlung von geerbten in schulisches Kapital durch die Bestätigung der Schule durch Zeugnisse etc. (vgl. Bourdieu 1982, S. 143 und 2006, S. 14-52) kann von einer Weitergabe habitueller Muster gesprochen werden. Ohne entsprechende Förderung und die Erweiterung der Möglichkeiten, die der eigene 
Habitus eingrenzt, ist ein gesellschaftlicher 〈Aufstieg〉 nur unter enormen Anstrengungen vorstellbar.

\section{Das soziale Kapital}

«Das soziale Kapital besteht aus Möglichkeiten, andere um Hilfe, Rat oder Information zu bitten sowie aus den mit Gruppenzugehörigkeiten verbundenen Chancen, sich durchzusetzen» (Fuchs-Heinritz/König 2005, S. 166). Das soziale Netz einer Person ist deren soziales Kapital, auf das sie bei Bedarf zurückgreifen kann. Darunter fallen z. B. Freundschaften, Geschäftsverbindungen und Mitgliedschaften in Verbänden. Die Aufrechterhaltung und Reproduktion dieser Kontakte erfordert eine andauernde Beziehungsarbeit (vgl. Baumgart 2000, S. 226).

Je umfassender ein solches Netz an sozialen Beziehungen, das ein Akteur [...] aufrechterhält und das er im Bedarfsfall benutzen kann, gestaltet ist, desto grösser sind seine Profitchancen bei der Reproduktion seines ökonomischen und kulturellen Kapitals. (Schwingel 2005, S. 92)

Dabei erhöhen gegenseitige Anerkennung und Wertschätzung innerhalb der Beziehungen die Chance, durch Mitgliedschaften oder Unterstützungshandlungen Beistand bzw. Vorteile zu erzielen (vgl. Fuchs-Heinritz/ König 2005, S. 166). Der strategische Ausbau des Beziehungsnetzes wird dabei bewusst oder unbewusst zum Zweck des Erhalts eines späteren Nutzens vollzogen (vgl. Bourdieu 1983, S. 192). Der Nutzen hängt von der Kapitalausstattung der Personen und der Ausdehnung des Netzes ab:

Der Umfang des Sozialkapitals, das der einzelne besitzt, hängt demnach sowohl von der Ausdehnung des Netzes von Beziehungen ab, die er tatsächlich mobilisieren kann, als auch von dem Umfang des (ökonomischen, kulturellen oder symbolischen) Kapitals, das diejenigen besitzen, mit denen er in Beziehung steht. (Bourdieu 1983, S. 191)

Wie wichtig ein soziales Netz für das Medienhandeln sein kann, zeigt sich bei technischen oder auch inhaltlichen Problemen im Umgang mit der Technik: Es ist nicht immer notwendig medienkompetent zu sein, wenn entsprechend kundige Personen verfügbar sind und bei etwaigen diffizilen Angelegenheiten aushelfen (vgl. Kommer/Biermann 2005, S. 16). Aus dieser Sicht ist diese Kapitalsorte in ihrer Bedeutung nicht zu unterschätzen; besonders deutlich wird dies, wenn wir uns das Web 2.0 (social web) unter den Aspekten der Kollaboration vergegenwärtigen (vgl. Marotzki/Jörissen 2008).

Die individuelle Ausstattung mit Kapital ist elementarer Teil des Habitus. Dabei ist immer zu berücksichtigen, dass eine historische Sichtweise mit eingebunden werden muss: «Als Produkt der Geschichte produziert der Habitus individuelle und kollektive Praktiken, also Geschichte, nach den von der Geschichte erzeugten Schemata; er gewährleistet die aktive Präsenz früherer Erfahrungen, die sich in je- 
dem Organismus in Gestalt von Wahrnehmungs-, Denk- und Handlungsschemata niederschlagen [...]» (Bourdieu 1987, S. 101).

Für eine an medienpädagogischen Fragen orientierte Forschung bedeutet dies, dass die jeweilige Mediennutzung wie andere Dispositionen immer auf bereits gemachte Erfahrungen aufbaut, also in die Vergangenheit zurückreicht. Dies verlangt die Berücksichtigung des Herkunftsmilieus und des spezifischen Umfelds (Existenzbedingungen), die einen durchaus relevanten und beachtlichen Einfluss auf die Ausbildung habitueller Muster haben (vgl. Kommer 2006). Nach Bourdieu sind es vor allem die Klasse, das Geschlecht und das soziale Feld, die drei zentrale Strukturkategorien bilden. Anhand dieser kann der Habitus in seinem spezifischen sozialen Kontext betrachtet und analysiert werden (vgl. Krais/Gebauer 2002, S. $31 \mathrm{ff})$. Durch die Berücksichtigung des Herkunftsmilieus bzw. Bildungsmilieu gemessen am Schul- und Hochschulabschluss und sekundär am Beruf des Vaters (vgl. Bourdieu 1982, S. 34) und - wenn auch nicht immer im ausreichenden Umfang des Geschlechts werden zwei dieser Kategorien abgedeckt. Das soziale Feld oder auch der soziale Raum erhält in diesem Kontext eine besondere Bedeutung. Die Berücksichtigung dieser Aspekte stellt die Relationen, oder anders ausgedrückt die Beziehungen, zu anderen Mediennutzern her. Über diese Vorgehensweise können Ungleichheiten im besonderen Masse herausgearbeitet werden, da distinktive Aspekte des Habitus in der Praxis über Vergleiche bzw. Typisierungen sichtbar und erfassbar werden. Mit Hilfe dieses Raums und den darin existierenden Relationen werden Unterschiede greifbar und können beschrieben werden. Erst im Verhältnis zu anderen habituellen Mustern werden Differenzen und ihre Auswirkungen auf die Möglichkeiten und Grenzen individuellen Handelns deutlich. Damit wird nachvollziehbar, welche Barrieren strukturell (auf gesellschaftlicher Ebene) und individuell (anders ausgedrückt: in den Köpfen der Menschen) existieren und für die Aufrechterhaltung und Reproduktion dieser Unterschiede ausgemacht verantwortlich sind.

\section{Die Bedeutung des Kapitals für die Distinktion und die Reproduktion von Ungleichheiten}

Die Schule verstärkt neben der Familie mit ihrem spezifischen Milieu den Effekt der Separation noch weiter, indem sie bestimmte habituelle Muster verbunden mit einer bestimmten Kapitalausstattung fördert: «In Wirklichkeit jedoch vermittelt jede Familie ihren Kindern auf eher indirekten als direkten Wegen ein bestimmtes kulturelles Kapital und ein bestimmtes Ethos, ein System impliziter und tief verinnerlichter Werte, das u. a. auch die Einstellungen zum kulturellen Kapital und zur schulischen Institution entscheidend beeinflusst. Das kulturelle Erbe, das unter beiden Aspekten nach sozialen Klassen variiert, ist für die ursprüngliche Ungleichheit der Kinder in Bezug auf die schulische Bewährungsprobe und damit die unterschiedlichen Erfolgsquoten verantwortlich» (Bourdieu 2006, S. 26, Herv. im 
Original). Damit wird noch einmal hervorgehoben, dass Bildungsabschlüsse und die soziale Herkunft (insbesondere bei dem Erwerb der ersten Bildungsabschlüsse) die Akkumulation von kulturellem Kapital in besonderer Weise beeinflusst.

Die Kapitalausstattung repräsentiert als Teil der strukturierten Struktur die - wenn teilweise auch schwierig - messbaren Dispositionen. Die Akkumulation von Kapital beginnt bereits in frühsten Jahren und ist fundamental für die Ungleichheit der verschiedenen Herkunftsmilieus, die somit die Basis für unterscheidbares menschliches Agieren bilden. Die Individuen unterscheiden sich in sozialer Weise zunächst durch die Verfügung über das eigene Kapital (bzw. durch die individuelle Kombination dessen). Doch auch die relative Stellung der Akteure untereinander spielt dabei eine Rolle. Eine Frage soll die weitere Argumentation unterstützen: In welcher Beziehung stehen zwei Menschen und worin unterscheiden sie sich? Dies kann zwar aufgrund der unterschiedlichen Kapitalausstattung analysiert werden (anhand der «feinen Unterschiede»), aber erst mit einem genaueren Blick auf die Handlungen und Dinge im Verhältnis zueinander und untereinander innerhalb der Beziehung dieser beiden Personen werden die distinktiven (oder im Umkehrschluss die gemeinsamen) Muster deutlich. Der Zusammenhang von Herkunftsmilieu und Lebensführung ist fundamental für die Ausbildung unterschiedlicher habitueller Muster, die erst über einen Vergleich als unterschiedlich erkannt und wahrgenommen werden. «Die unterschiedlichen Praktiken, Besitztümer, Meinungsäusserungen erhalten ihren sozialen Sinn also dadurch, dass sie etwas anzeigen, soziale Unterschiede nämlich, die Zugehörigkeit zu der einen oder zu der anderen soziale Gruppe oder Klasse» (Krais/Gebauer 2002, S. 37).

Mit dem Anzeigen der Zugehörigkeit ist eine Klassifikation möglich: «Die vom Soziologen klassifizierten sozialen Akteure sind mithin Produzenten nicht nur von klassifizierbaren, sondern auch von klassifizierenden Akten, die ihrerseits klassifiziert sind» (Bourdieu 1982, S. 728). Sie sind vor allem deshalb klassifizierbar, weil der Habitus sich aufgrund von Erfahrungen bildet, die im Verlauf der individuellen «Sozialisation» gemacht werden. Diese Erfahrungen wirken handlungs- und wahrnehmungsleitend, d. h., dass neue Erfahrungen aufgrund vorhandener Schemata inkorporiert werden und somit an ältere Strukturen anknüpfen.

Da er ein erworbenes System von Erzeugungsschemata ist, können mit dem Habitus alle Gedanken, Wahrnehmungen und Handlungen, und nur diese, frei hervorgebracht werden, die innerhalb der Grenzen der besonderen Bedingungen seiner eigenen Hervorbringung liegen. Über den Habitus regiert die Struktur, die ihn erzeugt hat, die Praxis, und zwar nicht in den Gleisen eines mechanischen Determinismus, sondern über die Einschränkungen und Grenzen, die seinen Erfindungen von vornherein gesetzt sind. (Bourdieu 1987, S. 102-103)

Eine Klassifizierung beruht somit unter der Berücksichtigung der Existenzbedingungen auf beschreibenden Dualismen wie oben/unten, reich/arm, etc. (vgl. Bour- 
dieu 1982, S. 279). Bezogen auf die Mediennutzung sind die Gegensatzpaare in ihrer Relevanz nicht zu unterschätzen, wie das Beispiel von Mikos (2007; S. 55) zeigt: «Die Unterscheidung [öffentl.-rechtl. vs. privatrechtl. Rundfunk, Anm. Ralf Biermann] spielt auch in alltäglichen Geschmacksurteilen eine Rolle, wie z. B. die von Harald Schmidt ausgelöste Debatte über das «Unterschichtenfernsehen` gezeigt hat.» Das Fernsehen - und hier insbesondere das privatrechtliche - ist mit einer Abwertung verknüpft, die dazu führt, dass eine Nutzung stark distinktiv besetzt ist und sich Personen zugleich gedrängt fühlen sich davon abzugrenzen und somit gezwungen sind zu positionieren. ${ }^{3}$

Nach Mikos können die individuellen Zuordnungen anhand der unterschiedlichen Formen des öffentlich-rechtlichen und des privatrechtlichen Rundfunks somit klassifiziert werden und mit distinktiven Handlungen in Verbindung gebracht werden. Die Erforschung von Bewertungen ist natürlich keine neue Erfindung und kann bereits auch als lange etabliert bezeichnet werden wie z. B. in der Langzeitstudie Massenkommunikation. Dort finden sich durch die befragten Personen bewertete Images und Nutzungsmotive als Bewertungsgrundlage bezogen auf die beiden rechtlich unterschiedlichen Rundfunkformen dieser (vgl. Reitze/Ridder 2006, S. 237ff). Dabei bleibt jedoch der Aspekt der Distinktion meist unberücksichtigt. Auch wenn nicht jedes Handeln auf Distinktion ausgerichtet ist, so ist der individuelle Habitus doch anhand dieser Abgrenzungseffekte beschreibbar und bietet somit eine Möglichkeit der empirischen Zuordnung. Wichtiger aber noch ist, dass diese Verbindung den ersten Schritt zu einem tieferen Verständnis darstellt und den Zugriff auf den Raum der Möglichkeiten (und somit auch Grenzen) einer Person eröffnet, der von den Schemata des Habitus aufgespannt wird.

\section{Soziokulturelle Unterschiede erfassen}

Die dargelegten Bewertungsmuster anhand von Gegensatzpaaren machen deutlich, dass diese durchaus als Beurteilungsaggregat herangezogen werden können. Eine Verkürzung auf einzelne wenig basale, zu kurz greifende Dualismen macht dabei wenig Sinn, wenn man sich auf das Habitus-Konzept bezieht. Vielmehr ist es notwendig, mehrere auf einen Gegenstandsbereich bezogene Schemata und Strukturen des Habitus herauszuarbeiten, um diesen möglichst detailliert und genau beschreiben zu können. Dabei sollten diese im idealen Fall aufeinander bezogen sein und Strukturen niedrigeren und höheren Rangs kombinieren, die mit Hilfe «multivariabler Analysen durch Überkreuzen logisch permutabler Kriterien» (Bourdieu 1979, S. 189) zu einer genaueren Spezifikation gebracht werden können (vgl. Bourdieu 1979, S. 188-189).

Die Schwierigkeit liegt darin, die entsprechenden und anwendbaren Gegensatzpaare für Forschungszwecke herauszuarbeiten oder durch die Interviewpartner

Zugleich bedeutet dies nicht, dass diese Personen das Fernsehen nicht mehr nutzen. 
verbalisieren zu lassen. Durch die Inkorporierung habitueller Muster werden diese unbewusst und verschleiert, sodass sich ein Zugriff z. B. über Fragebogen oder Interview recht schwierig gestaltet. Es existieren durchaus geeignete Methoden (wie z. B. die dokumentarische Methode nach Bohnsack 2003), die explizit den modus operandi (also habituelle Muster) im Blickpunkt haben. Ebenso können im quantitativen Bereich Clusteranalysen helfen, habituelle Muster und Strukturen aufzudecken, wie dies z. B. von Treumann etal. (2007) in der Studie zum Medienhandeln Jugendlicher durchgeführt wurde.

Die gesamte forschende Tätigkeit, die Gegensatzpaare zu erarbeiten und in Verbindung mit spezifischen Abgrenzungsmustern zu bringen (also bei den sfeinen Unterschieden> stehen zu bleiben), bleibt für eine Erklärung soziokultureller Unterschiede in Hinblick auf Reproduktion und Barrieren immer noch unzureichend, wenn diese nicht in Verbindung mit den Existenzbedingungen der Akteure gebracht werden. Ohne diese Koppelung stünden diese für sich und die Verwobenheit mit dem Alltag, den spezifischen Lebensbedingungen und letztendlich mit den gesellschaftlichen Ursachen ginge verloren, was jedoch nicht bedeutet, dass letztendlich alle Aspekte der Lebenswelt eines Akteurs zu erfassen sind.

Letztlich bestimmen also der jeweilige Fokus der Analyse und die Feinheit des analytischen Rasters, welche Aspekte des habituellen Dispositionssystems in den Vordergrund einer sozialwissenschaftlichen Untersuchung treten: die individuellen (positions- oder laufbahnspezifischen) Merkmale oder die gesellschaftlichen, d. h. gruppen- und klassenspezifischen Bedingungen, die im Habitus ihren Niederschlag finden. (Schwingel 1995, S. 73)

Durch die Verbindung historisch gewachsener gesellschaftlicher Strukturen und individueller Merkmale können die Bedingungen und Muster soziokultureller Unterschiede herausgearbeitet werden, die fortgeführt ebenso im Bereich des Medienhandelns existieren. Unterschiedlich ausgeprägte Formen von Medienkompetenz können so auf ihre Genese vor dem Hintergrund historischer und spezifischer Existenzbedingungen im Rahmen des Habitus-Konzepts überprüft werden ${ }^{4}$. Dieser mediale Habitus (vgl. Kommer/Biermann 2007, S. 207 und Biermann 2009) bietet sich als theoretischer Bezugsrahmen für die Analyse von medienpädagogischen Fragestellungen an, da er das Medienhandeln in breiter Form unter Berücksichtigung der individuellen Lebenssituation und der Sozialisationserfahrungen erfasst. Problematisch ist dabei die methodische Vorgehensweise, da sich habituelle Muster aufgrund der unbewussten Form des Habitus oft dem Zugriff der Forscher entziehen. «Die jeweils neuen Mitglieder einer Sozialgruppe werden in einer Weise geformt, dass sie diese Formung vergessen, sich aber in vielfältiger Weise in der Lebensführung bedienen können» (Fuchs-Heinritz/König 2005, S. 136). Dadurch ist es für viele Interviewpartner schwierig die eigenen Schemata z. B. in Interviews zu

So zeigen z. B. die Arbeiten von Schäffer (2003) zu generationsspezifischen Medienpraxiskulturen wie unterschiedliche Generationen mit Medien umgehen und diese bewerten. 
explizieren. Aus diesem Grund ist es ratsam mit Hilfe einer Triangulation ( z. B. aus Interviews und Videobeobachtungen) zu arbeiten (vgl. Biermann/Kommer 2004) und damit einen tiefergehenden Blick aus verschiedenen Perspektiven auf die Dispositionen werfen zu können. Dabei ist es durchaus hilfreich auf die distinktiven Muster zurückzugreifen bzw. diese anzusprechen, um Reaktionen der Probanden hervorzurufen, die eine Klassifikation ermöglichen und die spezifischen Schemata greifbar machen. Durchaus muss man eingestehen, dass man in vielen Forschungsprojekten diese Komplexität nicht immer befriedigend einbeziehen kann. Besonders deutlich wird dies, wenn man das Werk «Das Elend der Welt» (Bourdieu 1997) betrachtet. Das methodische Vorgehen wird hier näher erläutert und reflektiert. Die Beschreibungen machen deutlich, dass für die Erhebung von Daten von Dispositionen, distinktiven Mustern und spezifischen Existenzbedingungen (inklusive der Ursachen für die Ungleichheiten) ein feinfühliges methodisches Vorgehen notwendig ist.

\section{Ausblick}

Der Habitus, die mit ihm in enger Weise verbundene Kapitalausstattung und die zugehörigen Reproduktionsmechanismen können eine nützliche Basis für medienpädagogische Arbeiten zum Verständnis von soziokulturellen Unterschieden sein. Dem vorausgesetzt aber sind die folgenden drei Prämissen:

Zunächst ist es durch schnelle technologische und gesellschaftliche Wandlungsprozesse unablässig notwendig, entsprechende Erkenntnisse zu generieren, die Erklärungen für die Ausbildung bestimmter medialer Habitus und deren für die Praxis relevanten Mechanismen liefern. Letztere wandeln und transformieren sich immer stärker durch sich verändernde Existenzbedingungen wie z. B. das sich ständig variierende Medienangebot. Damit erhält die Medienbiografie einen zentralen Stellenwert bei der Betrachtung soziokultureller Unterschiede bei der Betrachtung unterschiedlichen Medienhandelns.

Damit in engster Weise verbunden ist die Beachtung methodologischer Fragen, die den Zugang zu diesen häufig unbewussten und damit schwer erreichbaren Daten sicherstellen sollen (vgl. hierzu insbesondere Bourdieu 1997). Eine Berücksichtigung dieser forschungsrelevanten (und eigentlich bekannten) Aspekte bei der Erforschung soziokultureller Unterschiede erfolgt nur selten in ausreichender Form.

Sind entsprechend Erkenntnisse gewonnen, so gilt es diese in die medienpädagogische Praxis zu übertragen, um in adäquater Weise professionelles pädagogisches Handeln zu ermöglichen. Das bedeutet für die zielgruppenorientierte Medienarbeit nicht nur an den Interessen des Klientels anzusetzen und somit das kulturelle Kapital in den möglichen Grenzen des jeweiligen Habitus zu erweitern, sondern auch auszufächern und einen grösseren Variantenreichtum zu erreichen, um die Reproduktionsmechanismen sozialer Ungleichheit abzuschwächen. Dies setzt vo- 
raus, dass die Lernsettings verstärkt auch andere Weltsichten berücksichtigen, die ein Verstehen ‘fremder habitueller Welten ermöglichen. Kurz gesagt, werden so Erfahrungsräume geschaffen, die ausserhalb bisheriger Existenzbedingungen liegen. Zugleich erscheint es notwendig, dass sich auch die medienpädagogischen Fachkräfte ihres eigenen Habitus bewusst werden und diesen reflektieren (vgl. Kommer [im Druck]).

\section{Literatur}

Aufenanger, Stefan (1999a): Medienpädagogische Projekte - Zielstellungen und Aufgaben. In: Baacke, Dieter; Kornblum Susanne; Lauffer, Jürgen; Mikos, Lothar; Thiele, Günter A. (Hrsg.): Handbuch Medien: Medienkompetenz. Modelle und Projekte. Bonn: Bundeszentrale für politische Bildung, S. 94-97.

Aufenanger, Stefan (1999b): Medienkompetenz oder Medienbildung? Wie die neuen Medien Erziehung und Bildung verändern. In: Bertelsmann Briefe, H. 142, S. 21-24. Online verfügbar unter http://www.medienpaed.fb02.uni-mainz.de/stefan2005//Publikationen/PDF/aufenanger_medienkompetenz_medienbildung_99.pdf, zuletzt geprüft am 07.08.2008.

Baacke, Dieter (1973): Kommunikation und Kompetenz. Grundlegung einer Didaktik der Kommunikation und ihrer Medien. München: Juventa.

Baacke, Dieter (1999): Medienkompetenz als zentrales Operationsfeld von Projekten. In: Baacke, Dieter; Kornblum, Susanne; Lauffer, Jürgen; Mikos, Lothar; Thiele, Günter A. (Hrsg.): Handbuch Medien: Medienkompetenz. Modelle und Projekte. Bonn: Bundeszentrale für politische Bildung, S. 31-35.

Bauer, Ullrich (2002): Selbst- und/oder Fremdsozialisation: Zur Theoriedebatte in der Sozialisationsforschung. In: ZSE Zeitschrift für Soziologie der Erziehung und Sozialisation, Jg. 22, H. 2, S. 118-142.

Baumgart, Franzjörg (1997): Theorien der Sozialisation. Erläuterungen - Texte - Arbeitsaufgaben. 2. Aufl. Bad Heilbrunn: Klinkhardt, 2000.

Biermann, Ralf; Kommer, Sven (2004): Triangulation zur Annäherung an die Medienbiografie und die Mediennutzung von Jugendlichen. In: Buchen, Sylvia; Helfferich, Cornelia; Maier, Maja S. (Hrsg.): Gender methodologisch. Empirische Forschung in der Informationsgesellschaft vor neuen Herausforderungen. Wiesbaden: VS Verlag für Sozialwissenschaften, S. 195-211.

Biermann, Ralf (2009): Der mediale Habitus von Lehramtsstudierenden. Eine quantitative Studie zum Medienhandeln angehender Lehrpersonen. Wiesbaden: VS Verlag für Sozialwissenschaften.

Bohnsack, Ralf (2003): Rekonstruktive Sozialforschung. Einführung in qualitative Methoden 5. Auflage. Opladen: Leske + Budrich.

Bourdieu, Pierre (1979): Entwurf einer Theorie der Praxis auf der ethnologischen Grundlage der kabylischen Gesellschaft. 1. Aufl. Frankfurt am Main: Suhrkamp.

Bourdieu, Pierre (1982): Die feinen Unterschiede. Kritik der gesellschaftlichen Urteilskraft. Sonderausgabe. Frankfurt: Suhrkamp.

Bourdieu, Pierre (1983): Ökonomisches Kapital, kulturelles Kapital, soziales Kapital. In: Kreckel, Reinhard (Hrsg.): Soziale Ungleichheiten. Göttingen: Schwartz (Soziale WeltSonderband, 2), S. 183-199. 
Bourdieu, Pierre (1987): Sozialer Sinn: Kritik der theoretischen Vernunft. Frankfurt am Main: Suhrkamp.

Bourdieu, Pierre (1997): Das Elend der Welt. Zeugnisse und Diagnosen alltäglichen Leidens an der Gesellschaft. Konstanz: UVK.

Bourdieu, Pierre (2003): In Algerien. Zeugnisse der Entwurzelung. Graz: Ed. Camera Austria.

Bourdieu, Pierre (2006): Wie die Kultur zum Bauern kommt. Über Bildung, Schule und Politik. Hamburg: VSA-Verlag.

Deutsches PISA-Konsortium (Hrsg.) (2005): PISA 2003. Der zweite Vergleich der Länder in Deutschland; was wissen und können Jugendliche. Münster: Waxmann.

Ditton, Hartmut (2007): Der Beitrag von Schule und Lehrern zur Reproduktion von Bildungsungleichheit. In: Becker, Rolf; Lauterbach, Wolfgang (Hrsg.): Bildung als Privileg. Wiesbaden: VS Verlag für Sozialwissenschaften, S. 243-271.

Friebertshäuser, Barbara; Rieger-Ladich, Markus; Wigger, Lothar (Hrsg.) (2006): Reflexive Erziehungswissenschaft. Forschungsperspektiven im Anschluss an Pierre Bourdieu. Wiesbaden: VS Verlag für Sozialwissenschaften I GWV Fachverlage GmbH Wiesbaden.

Fuchs-Heinritz, Werner; König, Alexandra (2005): Pierre Bourdieu. Eine Einführung. Konstanz: UVK.

Gapski, Harald (2001): Medienkompetenz. Eine Bestandsaufnahme und Vorüberlegungen zu einem systemtheoretischen Rahmenkonzept. 1. Aufl. Wiesbaden: Westdeutscher Verlag.

Groeben, Norbert (2002): Dimensionen der Medienkompetenz: Deskriptive und normative Aspekte. In: Groeben, Norbert; Hurrelmann, Bettina (Hrsg.): Medienkompetenz. Voraussetzungen, Dimensionen, Funktionen. Weinheim: Juventa-Verlag (Lesesozialisation und Medien), S. 160-197.

Kommer, Sven (2006): Zum medialen Habitus von Lehramtsstudierenden. Oder: Warum der Medieneinsatz in er Schule eine so «schwere Geburt ist. In: Treibel, Annette; Maier, Maja S.; Kommer, Sven; Welzel, Manuela (Hrsg.): Gender medienkompetent. [Medienbildung in einer heterogenen Gesellschaft]. Wiesbaden: VS Verlag für Sozialwissenschaften, S. 165-178.

Kommer, Sven (im Druck): Kompetenter Medienumgang? Eine qualitative Untersuchung zum medialen Habitus und zur Medienkompetenz von Schülerlnnen und Lehramtsstudierenden. Leverkusen: Verlag Barbara Budrich.

Kommer, Sven; Biermann, Ralf (2007): Zwischen Erinnerung und Inszenierung - Medienbiografien medial. In: Zentrum für Anthropologie und Gender Studies (Hrsg.): Erinnern und Geschlecht. Band II. Freiburg: Jos-Fritz-Verl. (Freiburger FrauenStudien, 20), S. 195-222.

Krais, Beate; Gebauer, Gunter (2002): Habitus. Bielefeld: transcript Verlag.

Kübler, Hans-Dieter (1999): Medienkompetenz - Dimensionen eines Schlagwortes. In: Schell, Fred; Stolzenburg, Elke; Theunert, Helga (Hrsg.): Medienkompetenz - Grundlagen und pädagogisches Handeln. München: kopaed, S. 25-47.

Liebau, Eckart (2006): Der Störenfried. Warum Pädagogen Bourdieu nicht mögen. In: Friebertshäuser, Barbara; Rieger-Ladich, Markus; Wigger, Lothar (Hrsg.): Reflexive Erziehungswissenschaft. Forschungsperspektiven im Anschluss an Pierre Bourdieu. Wiesbaden: VS Verlag für Sozialwissenschaften, S. 41-58.

Marotzki, Winfried; Jörissen, Benjamin (2008): Wissen, Artikulation und Biographie: theoretische Aspekte einer Strukturalen Medienbildung. In: Fromme, Johannes; Sesink, Werner (Hrsg.): Pädagogische Medientheorie. Wiesbaden: VS Verlag für Sozialwissenschaften, S. 51-70. 
Medienpädagogischer Forschungsverbund Südwest (2007a): KIM-Studie 2006 (Kinder und Medien, Computer und Internet). Basisuntersuchung zum Medienumgang 6- bis 13-Jähriger. Stuttgart: Medienpädagogischer Forschungsverbund Südwest.

Medienpädagogischer Forschungsverbund Südwest (2007b): JIM-Studie 2007 (Jugend, Information, (Multi-)Media). Basisuntersuchung zum Medienumgang 12- bis 19-jähriger. Stuttgart: Medienpädagogischer Forschungsverbund Südwest.

Mikos, Lothar (2007): Distinktionsgewinne - Diskurse mit und über Medien. In: Fromme, Johannes; Schäffer, Burkhard (Hrsg.): Medien - Macht - Gesellschaft. Wiesbaden: VS Verlag für Sozialwissenschaften, S. 45-60.

Moser, Heinz (2003): Von der Medienkompetenz zur Medienbildung. Diskurstheoretische Überlegungen. In: Medienwissenschaft Schweiz, H. 2, S. 26-34.

Niesyto, Horst (2007): Medienpädagogik, Mediensozialisation und soziale Benachteiligung. In: Kompetenzzentrum Informalle Bildung (Hrsg.): Grenzenlose Cyberwelt. Zum Verhältnis von digitaler Ungleichheit und neuen Bildungszugängen für Jugendliche. 1. Aufl. Wiesbaden: VS Verlag für Sozialwissenschaften, S. 153-174.

Otto, Hans Uwe; Kutscher, Nadia; Klein, Alexandra; Iske, Stefan (2005): Soziale Ungleichheit im virtuellen Raum: Wie nutzen Jugendliche das Internet. Erste Ergebnisse einer empirischen Untersuchung zu Online-Nutzungsdifferenzen und Aneignungsstrukturen von Jugendlichen. Online verfügbar unter http://www.bmfsfj.de/bmfsfj/generator/RedaktionBMFSFJ/Abteilung5/Pdf-Anlagen/jugend-internet-langfassung, property=pdf, bereic $h=$, sprache $=$ de, rwb=true.pdf, zuletzt aktualisiert am 17.9.2007.

Reitze, Helmut; Ridder, Christa-Maria (2006): Massenkommunikation VII. Eine Langzeitstudie zur Mediennutzung und Medienbewertung 1964-2005. Baden-Baden: Nomos (Schriftenreihe Media-Perspektiven, 19).

Schäffer, Burkhard (2003): Generationen - Medien - Bildung. Medienpraxiskulturen im Generationenvergleich. Opladen: Leske + Budrich.

Schell, Fred (1999): Aktive Medienarbeit mit Jugendlichen. Theorie und Praxis. 3. Aufl. München: kopaed (Reihe Medienpädagogik, Bd. 5).

Schorb, Bernd (1997): Medienkompetenz. In: Hüther, Jürgen; Schorb, Bernd; Brehm-Klotz, Christiane (Hrsg.): Grundbegriffe Medienpädagogik. München: kopaed. S. 234-240.

Schwingel, Markus (1995): Pierre Bourdieu zur Einführung. 5. verb. Auflage 2005. Hamburg: Junius.

Treumann, Klaus Peter; Meister, Dorothee M.; Sander, Uwe (2007): Medienhandeln Jugendlicher. Mediennutzung und Medienkompetenz; Bielefelder Medienkompetenzmodell. 1. Aufl. Wiesbaden: VS Verlag für Sozialwissenschaften. 